\title{
Bio-inspired radar: recognition of human echolocator tongue clicks signals
}

\begin{abstract}
Echolocation is a process where sound waves are transmitted and the echoes are analyzed to determine information about the surrounding environment. Principle of echolocation method inspire by bat have been widely used in Radar and Sonar application. What is less known, this technique also used by a small group of blind humans in their daily life mainly for navigation and object recognition with high accuracy. To date, only a few technical studies look at how these echolocators are able to detect their own echoes. The conventional detection using match filter like in Radar application for this signal is not suitable due to existence of multiple frequency components. Thus, this paper discusses an alternative approach to recognize human echolocator tongue click signals by using the Linde-Buzo-Gray Vector Quantization Method. The significant click features which is the multiple frequencies itself were extracted from the raw transmits and echo signal and were used for the recognition process. Although there are gaps still need to be filled, the biologically-inspired technique presented here may provide useful information particular in signal processing for radar and sonar systems in the future.
\end{abstract}

Keyword: Echolocation; Bio-inspired 